\title{
Influencia de los tiempos de respuesta prehospitalarios en la supervivencia de los pacientes politraumatizados en Navarra
}

\author{
Influence of prehospital response times in the survival \\ of trauma patients in Navarre
}

\author{
B. Ali Ali' ${ }^{1}$ M. Fortún Moral $^{2}$, T. Belzunegui Otano ${ }^{3}$, R. Teijeira Álvarez ${ }^{4}$, D. Reyero Díez \\ A. Cabodevilla Górriz ${ }^{3}$
}

\section{RESUMEN}

La relación entre los tiempos de respuesta y la mortalidad de los pacientes politraumatizados en la denominada "hora de oro" sigue siendo tema de debate.

El objetivo del presente estudio es determinar las variables relacionadas con la mortalidad en dichos pacientes y la influencia de los tiempos de respuesta de los Servicios Médicos de Emergencia en dicha mortalidad.

Para ello se analizaron los datos del Registro "Major Trauma de Navarra" (cohorte retrospectiva de pacientes politraumatizados atendidos por el sistema sanitario de Navarra) durante los cuatro años comprendidos entre 2010 y 2013.

De los 217 casos de trauma disponibles para el análisis, fallecieron 42 (19\%). En el análisis multivariante no se encontró asociación significativa entre los diferentes tiempos de respuesta y la mortalidad: llegada a la escena (odds ratio (OR) 1,0; intervalo de confianza al $95 \%$ (IC) de 0,99 a 1,01), en el escenario (OR 1,00; IC $95 \%$ de 0,98 a 1,02) y tiempo total (OR 1,00; IC $95 \%$ de 0,99 a $1,01)$. Las variables que influyen en la mortalidad son la edad del paciente y la gravedad de las lesiones medidas por el Triage-Revised Trauma Score (T-RTS) prehospitalario y el New Injury Severity Score (NISS).

La mortalidad de los pacientes politraumatizados atendidos por el sistema de emergencias en nuestra región está influida por la edad, y por la intensidad de la agresión sufrida determinada por el T-RTS prehospitalario y por el NISS. Los tiempos de respuesta prehospitalarios no influyen significativamente.

Palabras clave. Mortalidad del politraumatizado. "Hora de oro". Tiempos de respuesta prehospitalarios.

\begin{abstract}
The relation between response times and mortality of polytrauma patients in the so-called "golden hour" continues to be a subject of debate.

The purpose of this study is to determine the variables related to mortality in these patients and the influence of response times of the Emergency Medical Services in this mortality.

To this end, the data in the "Major Trauma of Navarre" Register (retrospective cohort of polytrauma patients attended to by the Navarre Health Service) were analyzed for the four year period between 2010 and 2013.

Of the 217 trauma cases available for the analysis, $42(19 \%)$ died. No significant association was found in the multivariate analysis between the different response times and mortality: arrival at the scene (odds ratio (OR) $1.0 ; 95 \%$ confidence interval (CI) from 0.99 to 1.01 ), in the scenario (OR $1.00 ; 95 \% \mathrm{CI}$ from 0.98 to 1.02 ) and total time (OR 1.00; $95 \%$ CI from 0.99 to 1.01 ). The variables that influenced mortality are patient age and severity of injuries measured by the prehospital TriageRevised Trauma Score (T-RTS) and the New Injury Severity Score (NISS)

The mortality of polytrauma patients attended to by the emergency system in our region is influenced by age and by the intensity of the aggression suffered, determined by the prehospital T-RTS and by the NISS. The response times of the hospital do not have a significant influence.
\end{abstract}

Keywords. Polytrauma mortality. "Golden hour". Prehospital response times.
1. Servicio de Urgencias del Complejo Hospitalario de Navarra y Centro de salud Rochapea.

2. Servicio de Urgencias del Hospital de Tudela.

3. Servicio de Urgencias del Complejo Hospitalario de Navarra.

4. Instituto Navarro de Medicina Legal.

5. Servicio Navarro de Salud. UVI-móvil.

\section{Correspondencia:}

Tomás Belzunegui Otano

Servicio de Urgencias

Complejo Hospitalario de Navarra

31008 Pamplona

E mail: tomas.belzunegui.otano@cfnavarra.es

Recepción: 15 de enero de 2015

Aceptación provisional: 13 de abril de 2015

Aceptación definitiva: 20 de abril de 2015 


\section{INTRODUCCIÓN}

Hoy en día continúa el debate sobre qué estrategia de actuación prehospitalaria es mejor en el tratamiento del paciente politraumatizado (PPT) si "cargar y correr" o "quedarse y estabilizar", y en este sentido siguen publicándose artículos que dan argumentos en uno u otro sentido ${ }^{1-6}$. La clave de este tema es conocer si el valor añadido que da la actuación in situ de los Servicios Médicos de Emergencia (cateterizar una vía en la escena del accidente o actuar sobre la vía aérea, inmovilizar o realizar cualquier otra acción sobre el PPT) a pesar de alargar los tiempos en escena se ve recompensado con una mayor supervivencia.

El objetivo del presente estudio es determinar las variables relacionadas con la mortalidad en dichos pacientes y la influencia de los tiempos de respuesta de los Servicios Médicos de Emergencia en dicha mortalidad.

\section{PACIENTES Y MÉTODO}

Este estudio se llevó a cabo en Navarra, región situada al norte de España y que limita con Francia, con una superficie de $10.421 \mathrm{Km}^{2}$ y 637.000 habitantes. El sistema de emergencias es gestionado por un Centro de Coordinación que moviliza los recursos prehospitalarios según la gravedad de las víctimas (ambulancias medicalizadas y no medicalizadas) que trasladan a los PPT a los correspondientes servicios de urgencias hospitalarios. La comunidad cuenta con un hospital terciario y dos hospitales generales comarcales.

Desde 2010 nuestra comunidad cuenta con el Major Trauma Registry de Navarra $(\mathrm{MTRN})^{7}$ que recoge retrospectivamente la cohorte de PPT gestionados por el sistema de emergencias con las variables definidas según el estilo normalizado Utstein y que se muestran en la tabla 1. Fueron incluidos todos los PPT lesionados por agentes externos de cualquier intencionalidad entre el 1 de enero de 2010 y el 31 de diciembre de 2013, con un New Injuri Severity Score (NISS) superior a $15 \mathrm{y}$ atendidos por las
UVI-Móviles del Sistema de Emergencias de Navarra. Fueron excluidos aquellos cuya admisión en el hospital se produjo tras más de 24 horas de sufrir la lesión, lesionados por asfixia por inmersión, lesionados por ahorcamiento o pacientes quemados que no presentaban otras lesiones traumáticas $^{8}$. El proceso utilizado para la recolección de datos ha sido descrito en detalle en una publicación previa9.

La protección de datos se garantizó con el uso de mecanismos de encriptación SSL 3.0/TLS 1.0 y registro de accesos. El Proyecto contó con el visto bueno del Comité ético de investigación clínica del Departamento de Salud del Gobierno de Navarra.

Los tiempos de respuesta fueron calculados automáticamente por la base de datos a través de las horas reflejadas en las aplicaciones informáticas del Sistema de Emergencias que gestiona los vehículos de atención prehospitalaria y la aplicación informática de los hospitales que gestiona los pacientes desde el momento que llegan al hospital. Los diferentes intervalos se obtuvieron en base a los tiempos: hora de llamada, hora de llegada a la escena, hora de salida de la escena y hora de llegada al hospital? .

Los datos categóricos se presentaron mediante el número absoluto y el porcentaje. Los datos cuantitativos se expresaron mediante la media y desviación estándar (SD) y la mediana y rango inter cuartil (IQR) cuando se consideró adecuado. Los datos categóricos se compararon mediante la prueba de $\chi 2$. Cuando no se cumplían las condiciones de aplicación, y en tablas $2 \times 2$, se utilizó el test exacto de Fisher. Las variables cuantitativas se compararon mediante el test de la $t$ de Student y las pruebas no paramétricas mediante la prueba de la U de Mann-Whitney. Se utilizó la regresión logística para evaluar la asociación entre los tiempos de respuesta con la mortalidad con control de las variables que influyen en la misma. La variable dependiente fue la supervivencia o no y las variables independientes aquellas que resultaron significativas en el análisis bivariante y los tiempos de respuesta de atención prehospitalaria, objeto del presente estudio. 
Tabla 1. Variables de los pacientes incluidos en la base con sus correspondientes categorías

\begin{tabular}{|c|c|}
\hline $\begin{array}{l}\text { Variables relacionadas con la } \\
\text { fragilidad del paciente }\end{array}$ & Categorías \\
\hline Edad & Edad del paciente en el momento del accidente \\
\hline Sexo & $1=$ hombre $/ 2$ = mujer \\
\hline $\begin{array}{l}\text { Morbilidad previa al accidente } \\
\text { según el sistema de clasificación } \\
\text { ASA-PS }\end{array}$ & $\begin{array}{l}1 \text { = sin patología } / 2=\text { enfermedad sistémica moderada } / 3=\text { enfermedad } \\
\text { sistémica grave }\end{array}$ \\
\hline $\begin{array}{l}\text { Variables relacionadas con el } \\
\text { accidente }\end{array}$ & Categorías \\
\hline Tipo predominante & $1=$ contuso $/ 2=$ penetrante \\
\hline Mecanismo & $\begin{array}{l}1=\text { vehículo de motor } / 2=\text { motocicleta } / 3=\text { bicicleta } / 4=\text { atropello } / 5=\text { otros } \\
\text { relacionados con tráfico } / 6=\text { arma de fuego } / 7=\text { arma blanca } / 8=\text { objetos } \\
\text { diversos } / 9=\text { caída de baja energía } / 10=\text { caída de alta energía }\end{array}$ \\
\hline Intencionalidad & 1 = accidental $/ 2$ = autoagresión $/ 3$ = agresión $/ 4$ = Otros \\
\hline Revised Trauma Score (RTS) & Recogido por los primeros intervinientes en el lugar del accidente \\
\hline Abbreviated Injury Scale (AIS) & Códigos AIS que reflejan la severidad de las lesiones del paciente \\
\hline $\begin{array}{l}\text { Injury Severity Score (ISS) } \\
\text { y New ISS (NISS) }\end{array}$ & Valores del ISS y NISS que reflejan la severidad de las lesiones \\
\hline $\begin{array}{l}\text { Variables relacionadas con la } \\
\text { atención pre hospitalaria }\end{array}$ & Categorías \\
\hline $\begin{array}{l}\text { Tiempo desde la alarma a la } \\
\text { llegada al escenario }\end{array}$ & $\begin{array}{l}\text { Tiempo trascurrido entre la entrada de la llamada de socorro al } 112 \text { y la } \\
\text { llegada a la escena de los recursos asistenciales extra hospitalarios }\end{array}$ \\
\hline Tiempo en el escenario & $\begin{array}{l}\text { Tiempo que trascurre desde la llegada del equipo al escenario hasta que } \\
\text { sale hasta el hospital }\end{array}$ \\
\hline $\begin{array}{l}\text { Tiempo desde la alarma a la } \\
\text { llegada al hospital }\end{array}$ & $\begin{array}{l}\text { El tiempo entre la entrada de la llamada de alarma al } 112 \text { hasta que el } \\
\text { paciente llega al hospital }\end{array}$ \\
\hline $\begin{array}{l}\text { Nivel de los primeros } \\
\text { intervinientes }\end{array}$ & $\begin{array}{l}1 \text { = nivel I/ sin cuidados/ } 2 \text { = nivel II/ Soporte Vital Básico/ } 3 \text { = nivel III/ } \\
\text { soporte vita intermedio (presencia de recursos de Atención Primaria)/ } \\
4 \text { = nivel IV/ Soporte vital Avanzado (UVI-Móvil o helicóptero medicalizado }\end{array}$ \\
\hline Intubación pre hospitalaria & $1=\mathrm{si} / 2$ = no \\
\hline Resultado & Categorías \\
\hline Destino al alta & $\begin{array}{l}1=\text { domicilio } 2=\text { rehabilitación } / 3=\text { Fallecido } / 4=\text { Traslado a otro centro de } \\
\text { mayor nivel } / 5=\text { hospital de larga estancia }\end{array}$ \\
\hline $\begin{array}{l}\text { Secuelas a su alta hospitalaria } \\
\text { según escala de Glasgow }\end{array}$ & $\begin{array}{l}1=\text { sin secuelas } / 2 \text { = secuelas moderadas } / 3=\text { grandes secuelas con gran } \\
\text { dependencia } / 4=\text { estado vegetativo } / 5=\text { muerte }\end{array}$ \\
\hline Supervivencia & 1 = fallecimiento $/ 2$ = supervivencia (a los 30 días) \\
\hline
\end{tabular}

\section{RESULTADOS}

Se incluyeron para el análisis los datos de 462 PPT que cumplieron los criterios de inclusión. De ellos fueron excluidos 215 casos por ausencia de alguno de los tiempos de respuesta (escena o tiempo de transporte); 8 por tiempos erróneos (tiempos me- nores de cero o mayores de 300 minutos); 2 casos por ausencia de variable dependiente (supervivencia o exitus); 2 por ausencia de NISS; 15 por ausencia de T-RTS y 3 por no constar la edad. Después de todas las exclusiones se dispuso de 217 pacientes (Fig.1). 


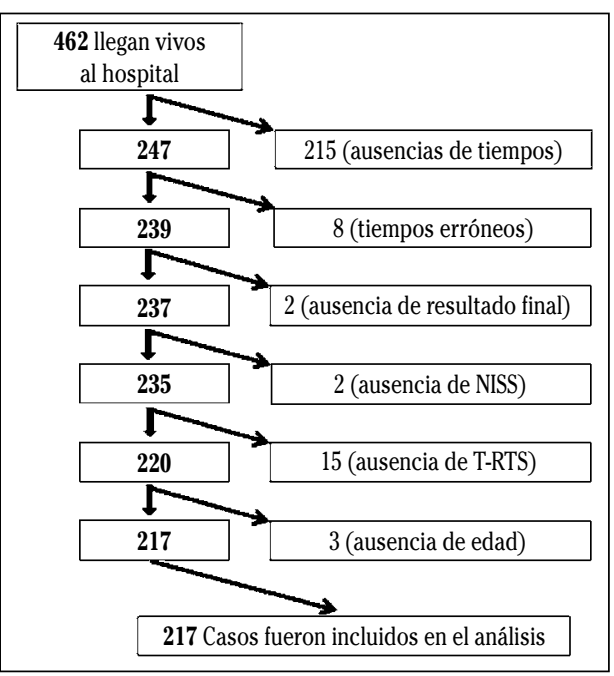

Figura 1. Diagrama de flujo. Pacientes incluidos en el estudio.

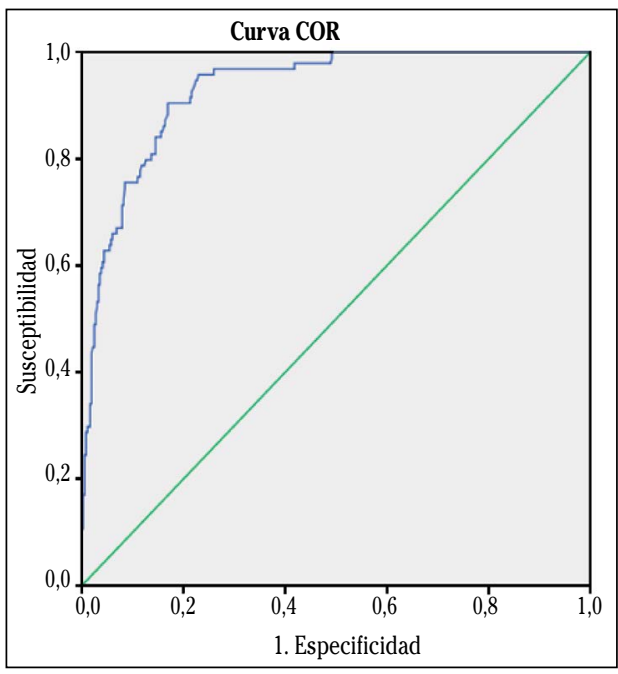

Figura 3. Curva ROC de los factores asociados a la mortalidad de los pacientes politraumatizados del Modelo 1, Área bajo la curva/ 0,931 IC 95\% 0,873-0,964.

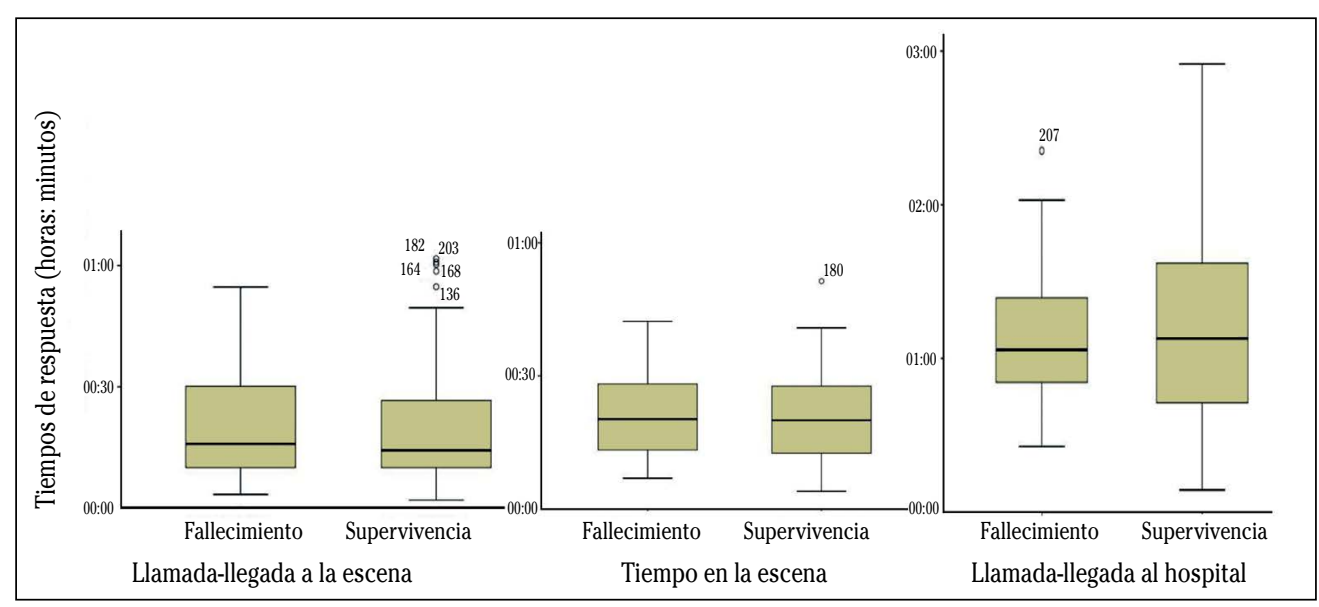

Figura 2. Diagramas de cajas en las que se observan los tiempos de respuesta de los equipos de emergencias desglosados en los grupos de pacientes que fallecieron y sobrevivieron,

De los 217 casos de PPT disponibles para el análisis, fallecieron 42 (19\%). Del total de las lesiones 206 (94\%) fueron contusas 42 fallecimientos (20\%) y $11(5 \%)$ fueron penetrantes, sin que se produjera ninguna defunción. La edad media y sexo de los accidentados fue de $50 \pm 21$ años $(69 \%$ hombres) con un rango entre 1 y 93 años.
Fueron intubados 35 pacientes (16\%), inmovilizados con diferentes métodos 141 (65\%); a 137 (63\%) se les cateterizó una vía periférica y a 145 (67\%) se les administró oxígeno por diferentes dispositivos.

La puntuación media y su correspondiente desviación estándar en los diferentes parámetros de gravedad de las lesiones 
Tabla 2. Distribución de los pacientes por las diferentes variables y resultado final

\begin{tabular}{|c|c|c|c|c|}
\hline Variables & Valores & Fallecidos & Supervivientes & $\mathbf{p}$ \\
\hline Total de pacientes & 217 & $42(19 \%)$ & $175(81 \%)$ & \\
\hline \multicolumn{5}{|l|}{ Edad } \\
\hline Media (desviación Estandar) & $50 \pm 21,3$ & $66,3 \pm 18,1$ & $46,1 \pm 20,2$ & $<0,01$ \\
\hline \multicolumn{5}{|l|}{ Sexo } \\
\hline Varones & $150(69,1 \%)$ & $27(18 \%)$ & $123(82 \%)$ & 0,46 \\
\hline Mujeres & $67(30,9 \%)$ & $15(22,4 \%)$ & $52(77,6 \%)$ & \\
\hline \multicolumn{5}{|l|}{ Tipo de lesión } \\
\hline Contusa & $206(94,1 \%)$ & $42(20,4 \%)$ & $164(79,6 \%)$ & 0,08 \\
\hline Penetrante & $11(5,1 \%)$ & $0(0 \%)$ & $11(100 \%)$ & \\
\hline \multicolumn{5}{|l|}{ Mecanismo } \\
\hline Trafico & $107(49,3 \%)$ & $17(15,9 \%)$ & $90(84,1 \%)$ & $\mathbf{0 , 0 3}$ \\
\hline Arma blanca o de fuego & $8(3,7 \%)$ & $0(0 \%)$ & $8(100 \%)$ & \\
\hline Caída & $49(22,6 \%)$ & $16(32,7 \%)$ & $33(67,3 \%)$ & \\
\hline Precipitación de altura & $36(16,6 \%)$ & $8(22,2 \%)$ & $28(77,8 \%)$ & \\
\hline Otros & $17(7,8 \%)$ & $1(5,9 \%)$ & $16(94,1 \%)$ & \\
\hline \multicolumn{5}{|l|}{ Intencionalidad } \\
\hline Accidental & $194(89,4 \%)$ & $36(18,6 \%)$ & $158(81,4 \%)$ & 0,56 \\
\hline Autoinflingida & $13(6 \%)$ & $4(30,8 \%)$ & $9(69,2 \%)$ & \\
\hline Agresión & $10(4,6 \%)$ & $2(20 \%)$ & $8(80 \%)$ & \\
\hline \multicolumn{5}{|l|}{ Índice fisiológico de gravedad } \\
\hline RTS en el lugar del accidente, media (DS) & $7 \pm 1,3$ & $5,8 \pm 1,8$ & $7,4 \pm 0,8$ & $<0,01$ \\
\hline \multicolumn{5}{|l|}{ Índice anatómico de gravedad } \\
\hline NISS, media (DS) & $29,1 \pm 10,8$ & $39,4 \pm 12,2$ & $26,6 \pm 8,8$ & $<0,01$ \\
\hline ISS & $21,7 \pm 9,5$ & $29,2 \pm 9,8$ & $19,9 \pm 8,6$ & $<0,01$ \\
\hline \multicolumn{5}{|l|}{ Intubación prehospitalaria } \\
\hline No & $182(83,9 \%)$ & $24(13,2 \%)$ & $158(86,8 \%)$ & $<0,01$ \\
\hline Sí & $35(16,1 \%)$ & $18(51,4 \%)$ & $17(48,6 \%)$ & \\
\hline \multicolumn{5}{|l|}{ Primer hospital de asistencia } \\
\hline Centro de Trauma & $155(71,4 \%)$ & $33(21,3 \%)$ & $122(78,7 \%)$ & 0,34 \\
\hline Hospital Comarcal & $62(28,6 \%)$ & $9(14,5 \%)$ & $53(85,5 \%)$ & \\
\hline \multicolumn{5}{|l|}{ Tiempos de Respuesta } \\
\hline Llamada - llegada a escena, media (SD) & $00 / 26 \pm 00 / 28$ & $00 / 21 \pm 00 / 15$ & $00 / 23 \pm 00 / 22$ & 0,68 \\
\hline Mediana (IQR) & $00 / 17(00 / 10-00 / 30)$ & $00 / 17 \overline{(00 / 13-00 / 31)}$ & $00 / 18 \overline{(00 / 10-00 / 34)}$ & 0,84 \\
\hline Tiempo en escena & $00 / 23 \pm 00 / 17$ & $00 / 22 \pm 00 / 15$ & $00 / 23 \pm 00 / 18$ & 0,33 \\
\hline \multirow[t]{3}{*}{ Llamada - llegada al hospital } & $00 / 21(00 / 12-00 / 30)$ & $00 / 17(00 / 14-00 / 31)$ & $00 / 17(00 / 13-00 / 31)$ & \\
\hline & $01 / 14+00 / 42$ & $01 / 08 \pm 00 / 28$ & $01 / 15+00 / 44$ & \\
\hline & $01 / 05(00 / 43-01 / 36)$ & $01 / 03(00 / 50-01 / 19)$ & $01 / 05(00 / 44-01 / 39$ & \\
\hline
\end{tabular}

fue en la escala de Glasgow $12+5$ puntos, RTS $11+1,3$ puntos y el NISS de $29+10$.

La variable dependiente fue la supervivencia a los 30 días, y en relación con esta variable, los cruces de las posibles variables predictoras asociadas con la misma, quedan reflejados en la tabla 2 .

Los tiempos de respuesta desglosados por pacientes que sobreviven o fallecen se reflejan en la Fig.2.
El análisis de regresión logística incluyendo las variables predictoras que resultaron significativas en el análisis bivariante y los tiempos de respuesta quedan reflejados en la tabla 3.

La precisión del modelo 1 queda representado por la curva Receiver Operating Characteristic (ROC) y el área comprendida bajo la misma que es del $93 \%$ queda reflejado en la Fig. 3. 
Tabla 3. Regresión logística de los factores independientes asociados a la mortalidad de los politraumatizados, Variable dependiente (fallecimiento $=1 /$ supervivencia $=0$ ), OR con sus correspondientes intervalos de confianza al $95 \%$,

\begin{tabular}{lccc}
\hline Modelo 1. Variables cuantitativas & OR & IC 95\% & p \\
\hline Edad & 1,11 & $1,03-1,19$ & 0,006 \\
\hline RTS prehospitalario & 0,36 & $0,17-0,78$ & 0,010 \\
\hline NISS & 1,20 & $1,06-1,35$ & 0,003 \\
\hline Intervalo 1/ llamada-llegada al escenario & 1,00 & $0,99-1,01$ & 0,926 \\
\hline Intervalo 2/ llegada-salida del escenario & 1,00 & $0,98-1,02$ & 0,892 \\
\hline Intervalo 3/ llamada-llegada a Urgencias & 1,00 & $0,99-1,01$ & 0,624 \\
\hline & & & \\
\hline Modelo 2. Variables cualitativas dicotómicas & $\mathbf{O R}$ & $\mathbf{I C ~ 9 5 \%}$ & $\mathbf{p}$ \\
\hline Edad & 4,47 & $2,43-7,55$ & 0,003 \\
\hline RTS prehospitalario & 16,00 & $10,51-21,03$ & 0,000 \\
\hline NISS & 12,56 & $9,31-17,45$ & 0,001 \\
\hline Intervalo 1/ llamada-llegada al escenario & 1,00 & $0,91-1,02$ & 0,835 \\
\hline Intervalo 2/ llegada-salida del escenario & 1,00 & $0,93-1,03$ & 0,820 \\
\hline Intervalo 3/ llamada-llegada a Urgencias & 1,00 & $0,85-1,07$ & 0,532 \\
\hline
\end{tabular}

Los ajustes se han realizado en el Modelo 1 por las variables cuantitativas/ edad, RTS prehospitalario y NISS que son aquellas que se han seleccionado entre las que presentaban significación estadística en el análisis bivariante y coherencia desde el punto vista clínico y de gestión del paciente, Se incluyen los tiempos de respuesta como variables de estudio, En el Modelo 2 se han incluido las mismas variables convertidas en dicotómicas/ edad (mayor o menor a 50 años)/ RTS (mayor o menor a 7)/ NISS (mayor o menor a 29)/ intervalo 1 (mayor o menor de $17 \mathrm{~min}$, )/ intervalo 2 (mayor o menor de $21 \mathrm{~min}$,)/ intervalo 3 (mayor o menor de $65 \mathrm{~min}$ ), Se han utilizado como punto de corte las medias en el caso de la edad, RTS y NISS y las medianas debido a la dispersión de los valores en los tiempos de respuesta.

\section{DISCUSIÓN}

Tal y como reconoce Prat en su publicación ${ }^{10}$, a pesar de ser el tiempo una pieza clave en un sistema de atención al trauma, el registro sistemático y el análisis de estos resultados son desconocidos en nuestro entorno, de ahí la importancia de difundir los resultados de los diferentes estudios que se llevan a cabo en nuestro país.

Las características de los PPT incluidos en nuestra base son muy parecidas a los encontrados en la base alemana Deutsche Gesellschaft für Unfallchirurgie (DGU) y otras de nuestro país coincidiendo plenamente en una edad media cercana a los 50 años, con un predominio de varones (70\%), con un tipo de lesiones casi totalmente contusas (solamente un $5 \%$ de lesiones penetrantes) y de tipo accidental. Casi la mitad de la casuística la aportan los accidentes de tráfico, seguidos por las caídas de baja energía (25\% de los casos) $)^{10-12}$.

En cuanto a las diferencias en nuestra base el Injury Severity Score (ISS) medio es de casi 22 mientras que en la base alemana es de 17. Es llamativa también una menor tasa de intubación prehospitalaria en nuestro caso ( $16 \%$ frente al $23 \%$ de la DGU) y una mayor mortalidad (19\% en Navarra frente al $10 \%$ de la DGU y Cataluña) ${ }^{10,12}$. No sabemos exactamente a qué obedecen dichas diferencias si bien una posibilidad es la inclusión en nuestra base de un grupo de pacientes ancianos con gran mortalidad y lesiones encefálicas severas a los que dada su situación de calidad de vida previa no se llega a intubar ni se realizan acciones agresivas. Se trata de pacientes ancianos frágiles, en tratamiento anticoagulante que su- 
fren caídas accidentales y que en muchas ocasiones acaban en fallecimiento ya que se limita el esfuerzo terapéutico ${ }^{7,9}$.

En nuestra base nos hemos ceñido estrictamente a los criterios de inclusión definidos por el estilo Utstein y pensamos que las diferencias con otros grupos pueden provenir de este grupo de pacientes que posiblemente no se incluyen en otras bases $^{8}$.

Los tiempos de respuesta obtenidos en nuestro estudio son muy similares a los obtenidos en otros estudios en países desarrollados y con características similares a los de nuestra región ${ }^{12-16}$. Un estudio francés que compara la gestión realizada en PPT por SAMU (atención medicalizada) con la filosofía de estabilización y traslado, frente a la realizada por bomberos de cargar y correr, muestra una mejor tasa de supervivencia de dichos pacientes a pesar de presentar índices de gravedad peores. En nuestra región, la gestión de los PPT se adapta a dicha filosofía de atención medicalizada y traslado y las conclusiones de su estudio serían aplicables a nuestro caso ${ }^{17}$. Los tiempos de respuesta son mayores en el caso de los pacientes trasladados por SAMU pero no influyen en la superviven$\mathrm{cia}^{18}$.

En la línea anteriormente expuesta, un estudio de Irán demuestra que la mortalidad se asoció con la gravedad de las lesiones y largos tiempos de transporte prehospitalario. En pacientes gravemente heridos que recibieron intervenciones de soporte vital avanzado se observaron tendencias de supervivencia positivas a pesar de aumentar los tiempos de transporte ${ }^{19}$.

En los estudios evaluados a nivel nacional, no se hace referencia a tiempos de respuesta, con lo que no existe un parámetro nacional con el que comparar nuestro estudio $^{10}$.

En cuanto a la influencia de los tiempos de respuesta en la supervivencia, diferentes estudios sugirieren que el aumento de los tiempos de respuesta prehospitalarios se asociaron con un aumento de la mortalidad de los PPT ${ }^{11,15}$, no obstante estas afirmaciones han sido contestadas en otras ocasiones por otros autores que ponen en tela de juicio este hallazgo y lo atribuyen a sesgos relacionados con muestras pequeñas de pacientes quirúrgicos altamente seleccionados, pacientes generados en áreas rurales con largos tiempos de respuesta o muestras mixtas que incluían pacientes con paro cardíaco no traumático ${ }^{20-22}$.

El término "hora de oro" es utilizado para identificar la necesidad de atención urgente de los PPT. Este concepto implica que la morbi-mortalidad se ve incrementada si el cuidado no se instaura en la primera hora después de la lesión y justifica la atención precoz de estos pacientes con los medios adecuados. Sin embargo, diferentes estudios reconocen que no hay datos objetivos que avalen dicho concepto ${ }^{6}$.

Algunos estudios centrados en pacientes muy específicos que se pueden beneficiar de intervenciones prehospitalarias "salvadoras" como las víctimas de trauma penetrante torácico, demuestran que los pacientes más graves llegaron a los centros de traumatología antes. La mortalidad estuvo fuertemente asociada a la gravedad de la lesión, y por ese motivo a pesar de los tiempos pre-hospitalarias más cortos se asociaron con una mayor supervivencia ${ }^{16}$. Como ya se ha dicho, en nuestra base las heridas penetrantes torácicas son muy escasas y difícilmente van a tener relevancia estadística por este motivo y por tanto tampoco los tiempos de respuesta prehospitalarios asociados a su tratamiento.

Una interesante revisión sistemática de 46 artículos demuestra que la mayoría de la investigación en trauma es favorable al traslado rápido sin actuaciones que puedan demorar su acceso al hospital en caso de trauma penetrante, y en casos de corta distancia a un hospital. En los pacientes con lesiones graves en la cabeza, el soporte vital avanzado proporcionado por los paramédicos y la intubación pueden ser perjudiciales. Si la atención prehospitalaria es proporcionada por un sistema medicalizado con experiencia, dichas intervenciones pueden ser beneficiosas para los pacientes con lesiones múltiples y lesiones cerebrales severas. Sin embargo reconoce que los resultados son contradictorios ${ }^{23}$. 
En un estudio con 3.656 pacientes, de los cuales fallecieron el $22 \%$, en el análisis multivariante tampoco se encontró asociación significativa entre el tiempo y la mortalidad por cualquiera de los intervalos (respuesta, en el lugar del siniestro, transporte y tiempo total ${ }^{14}$.

En relación con la denominada "hora de oro", diferentes autores reconocen que aunque hay pacientes gravemente lesionados, que pueden requerir actuaciones tiempo-dependientes para sobrevivir (desobstrucción de la vía aérea, ventilación, control de hemorragia externa en un sitio compresible, etc.), para la mayoría de los pacientes puede no haber efecto medible. También es plausible que la "hora de oro" dependa principalmente de la rapidez de las intervenciones en los hospitales (tiempo de realización de TAC o tiempo de primera intervención quirúrgica de control de daños, y por ello los tiempos de respuesta "per se", no entran en los análisis de regresión logística como variable relacionada con la supervivencia, como sucede en nuestro estudio.

Una línea interesante de investigación es la de algunos autores que critican el estudio de Newgard y col y piden que se den más datos de los 1.385 pacientes que murieron en la escena y fueron excluidos del estudio. Para estos pacientes se desconocía el intervalo de la intervención prehospitalaria y no se podía concluir que el tiempo no tuvo ningún efecto sobre su muerte ${ }^{24,25}$. En nuestro estudio tampoco contamos con tiempos de respuesta de estos pacientes, pero en la comparación que establecimos entre ambos grupos hemos demostrado que son varones en un porcentaje mayor, más jóvenes, con aumento de los sucesos autolíticos penetrantes posiblemente por armas de fuego y con una gravedad de las lesiones muy superior al otro grupo. $\mathrm{Pa}-$ rece razonable pensar que los tiempos de respuesta no son los responsables del fallecimiento de nuestros pacientes en la denominada "hora de oro", sino la gravedad de las lesiones.

Little afirma que más que en la "hora de oro", los proveedores de salud deben centrarse en la "oportunidad de oro" para proporcionar la mejor atención al paciente crítico controlando las situaciones que amenacen la vida del paciente y trasladándolo al centro apropiado en condiciones óptimas ${ }^{26}$.

En nuestro estudio, las diferencias en los tiempos de respuesta entre los pacientes que sobreviven y fallecen son muy pequeñas, incluso dándose la paradoja de que el tiempo total es menor en los que fallecen. Tal y como queda demostrado por la estadística multivariante esto se debe a la gravedad del paciente que hace que los sistemas prehospitalarios sean lógicamente más rápidos en estos pacientes ${ }^{16}$.

El estudio de la DGU sobre 15.103 pacientes presentó un tiempo en escena de 33 minutos (ligeramente superior a los nuestros) e identificó las intervenciones y las condiciones características con un impacto significativo en los tiempos en la escena del accidente. La intubación se asoció con tiempos más prolongados, mientras que la situación de coma se asoció con la reducción del mismo. Los tiempos totales son muy similares a los recogidos en nuestra serie y se encuentran en torno a los 60 minutos ${ }^{12}$.

En traumatismos craneales con AIS $>3$ se demostró aumento de la supervivencia en los pacientes que llegaron antes al hospital, pero el beneficio puede extenderse más allá de la "hora de oro". No hubo evidencia de mejores resultados en los pacientes que llegaron dentro de los primeros 60 minutos tras el accidente 4 .

El estudio tiene algunas limitaciones como ser una muestra de pacientes relativamente pequeña si se compara con las grandes bases internacionales. Asimismo hay una pérdida de 215 casos en los que no hay tiempos de respuesta y otros 8 erróneos, lo que puede dificultar el análisis de los resultados. Tampoco se incluyen en el análisis los pacientes fallecidos in situ y tal y como han comentado algunos autores, sería muy interesante conocer los tiempos de respuesta de los pacientes que no llegan vivos al hospital ${ }^{24,27}$. Dicho interrogante abre una nueva línea muy interesante en este tema.

Del estudio, concluimos que la mortalidad de los pacientes politraumatizados 
atendidos por el Sistema de Emergencias Médicas en nuestra región está influida por su fragilidad determinada por la edad y por la intensidad de la agresión sufrida medida por el Triage-Revised Trauma Score (T-RTS) y por el New Injury Severity Score (NISS). Los tiempos de respuesta prehospitalarios son suficientemente buenos como para no influir significativamente en el fallecimiento de dichos pacientes.

\section{BIBLIOGRAFÍA}

1. Tissier C, Bonithon-Kopp C, Freysz M. French Intensive care Recorded in Severe Trauma (FIRST) study group. Statement of severe trauma management in France; teachings of the FIRST study. Ann Fr Anesth Reanim 2013; 32: 465-471.

2. McCoy CE, Menchine M, Sampson S, Anderson C, KaHN C. Emergency medical services outof-hospital scene and transport times and their association with mortality in trauma patients presenting to an urban level I trauma center. Ann Emerg Med 2013; 61: 167-174.

3. Bartolacci RA, Munford BJ, Lee A, McDougall PA. Air medical scene response to blunt trauma: Effect on early survival. Med J Aust 1998; 169: 612-616.

4. Dinh MM, Bein K, Roncal S, Byrne CM, Petchell J, BRENNAN J. Redefining the golden hour for severe head injury in an urban setting: The effect of prehospital arrival times on patient outcomes. Injury 2013; 44: 606-610.

5. Feero S, Hedges JR, Simmons E, Irwin L. Does outof-hospital ems time affect trauma survival. Am J Emerg Med 1995; 13: 133-135.

6. Lerner EB, Moscati RM. The golden hour: Scientific fact or medical "urban legend"? Acad Emerg Med 2001; 8: 758-760.

7. Belzunegui T, Fortun M, Reyero D, Teijeira R. Implementation of the first spanish population- based register of multiple injury cases in Navarre. Emerg 2013; 25: 196-200.

8. Dick WF, Baskett PJ, Grande C, et al. Recommendations for uniform reporting of data following major trauma-the utstein style. an international trauma anaesthesia and critical care society (ITACCS) initiative. Br J Anaesth 2000; 84: 818-819.

9. Belzunegui T, Gradin C, Fortun M, Cabodevilla A, Barbachano A, Antonio Sanz J. Major trauma registry of Navarre (Spain)/ The accuracy of different survival prediction models. Am J Emerg Med 2013; 31: 1382-1388.
10. Prat S, Dominguez-Sampedro P, Koo M, Colilles C, Jimenez-Fabrega X, Espinosa L. TraumCat/ Preliminary analysis of a year of severe trauma registry in Catalonia. Emerg 2014; 26: 267 274.

11. Sampalis JS, Denis R, Lavoie A, et al. Trauma care regionalization/ A process-outcome evaluation. J Trauma 1999; 46: 565-579.

12. Wyen H, Lefering R, Maegele M, et al. The golden hour of shock - how time is running out/ Prehospital time intervals in Germany - a multivariate analysis of 15,103 patients from the TraumaRegister DGU(R). Emerg Med J 2013; 30: 1048-1055.

13. Carr BG, Caplan JM, Pryor JP, Branas CC. A meta-analysis of prehospital care times for trauma. Prehosp Emerg Care 2006; 10: 198206.

14. Newgard CD, Schmicker RH, Hedges JR, et al. Emergency medical services intervals and survival in trauma/ Assessment of the "golden hour" in a north american prospective cohort. Ann Emerg Med 2010; 55: 235-246.

15. Sampalis JS, Lavoie A, Williams JI, Mulder DS, KaLINA M. Impact of on-site care, prehospital time, and level of in-hospital care on survival in severely injured patients. J Trauma 1993; 34: 252-261.

16. Swaroop M, Straus DC, Agubuzu O, Esposito TJ, Schermer CR, Crandall ML. Pre-hospital transport times and survival for hypotensive patients with penetrating thoracic trauma. Emerg Trauma Shock 2013; 6: 16-20.

17. Gomez de Segura Nieva JL, Boncompte MM, Sucunza AE, Louis CL, Segui-Gomez M, Otano TB. Comparison of mortality due to severe multiple trauma in two comprehensive models of emergency care/ Atlantic pyrenees (France) and Navarra (Spain). J Emerg Med 2009; 37: 189-200.

18. Yeguiayan JM, Garrigue D, Binquet C, et al. Medical pre-hospital management reduces mortality in severe blunt trauma/ A prospective epidemiological study. Crit Care 2011; 15: R34.

19. Paravar M, Hosseinfour M, Mohammadzadeh M, MirzadeH AS. Prehospital care and in-hospital mortality of trauma patients in iran. Prehosp Disaster Med 2014; 29: 473-477.

20. Di Bartolomeo S, Valent F, Rosolen V, et al. Are pre-hospital time and emergency department disposition time useful process indicators for trauma care in italy? Injury 2007; 38: 305-311.

21. Ivatury RR, Nallathambi MN, Roberge RJ, RohMAN M, STAHL W. Penetrating thoracic injuries/ In-field stabilization vs. prompt transport. J Trauma 1987; 27: 1066-1073. 
22. Grossman DC, Kim A, Macdonald SC, Klein P, CoPASS MK, MAIER RV. Urban-rural differences in prehospital care of major trauma. J Trauma 1997; 42: 723-729.

23. Ryynanen OP, Iirola T, Reitala J, Palve H, MalMIVAARA A. Is advanced life support better than basic life support in prehospital care? A systematic review. Scand J Trauma Resusc Emerg Med 2010; 18: 18-62.

24. FleEt R, Poitras J. Have we killed the golden hour of trauma? Ann Emerg Med 2011; 57: 73-74.
25. Barrett TW, Brywczynski JJ, Schriger DL. Journal club/ Is the golden hour tarnished? registries and multivariable regression. Ann Emerg Med 2010; 55: 247-248.

26. LitTLE WK. Golden hour or golden opportunity/ Early management of pediatric trauma. Clin Pediatr Emerg Med 2010; 11: 4-9.

27. Alberdi F, Azaldegui F, Zabarte M, et al. Epidemiological profile of late mortality in severe polytraumatisms. Med Intensiva 2013; 37: 383-390. 